\title{
Light Localizations in Photonic Crystal Line Defect Waveguides
}

\author{
Toshihiko Baba, Member, IEEE, Daisuke Mori, Kyoji Inoshita, and Yusuke Kuroki
}

\begin{abstract}
In this paper, we discuss unique light localizations in photonic crystal line defect waveguides based on two different concepts. The first concept is an additional defect doping that breaks the symmetry of the line defect. Even though such a defect is open to the line defect, the optical field is well confined around the defect at cutoff frequencies of the line defect. This expands the design flexibility of microcavities and allows effective mode controls such as the single-mode operation. The lasing action of such cavities in a GaInAsP photonic crystal slab was experimentally observed by photopumping at room temperature. The second concept is a chirping of the waveguide structure. The photonic band of a waveguide mode has a band edge, at which the group velocity becomes zero. The band-edge condition shifts in a chirped line defect waveguide, so guided light reaches a zero group velocity point and is localized. A macroscopic behavior of this phenomenon was experimentally observed in a waveguide fabricated into a silicon-on-insulator substrate. In addition, a microscopic behavior was theoretically investigated, which suggested its applicability to a group delay device.
\end{abstract}

Index Terms-Chirped grating, defect laser, group delay device, group velocity, light localization, line defect waveguide, nanolaser, photonic crystal (PC).

\section{INTRODUCTION}

$\mathbf{P}$ HOTONIC crystals (PCs) are artificial multidimensional periodic structures with a period of the order of optical wavelength, which can be designed by the photonic band theory. One of the most unique properties of PCs is a photonic bandgap (PBG). It inhibits or restricts the existence of optical modes and allows the strong control of light emission and propagation. The most fundamental applications of the PBG are cavities including lasers [1]-[7] and waveguides [8]-[14], which are composed of point and line defects, respectively. Those formed into two-dimensional (2-D) PC slabs are now being studied worldwide. In these devices, the PBG acts as an angle-independent distributed Bragg reflector inside the 2-D plane and localizes or guides light in the defect region. To realize strong optical confinement in microcavities and microlasers, all sides of the point defect are normally surrounded by the PC to suppress the

Manuscript received December 2, 2003. This work was supported in part by the Nano-Photonic and Electron Devices Technology Project, Focused Research and Development Project for the Realization of the World's Most Advanced IT Nation, and the 21st COE Program for Creation of Future Social Infrastructure Based on Information Telecommunication Technology, all from the Ministry of Education, Culture, Sports, Science and Technology, and in part by the Japan Science and Technology Corporation under Core Research for Evolutional Science and Technology 530-13.

T. Baba, D. Mori, and Y. Kuroki are with the Department of Electrical and Computer Engineering, Yokohama National University, Yokohama 240-8501, Japan (e-mail: baba@ynu.ac.jp).

K. Inoshita is with the Sanyo Electric Co., Ltd., Osaka 574-8534, Japan. Digital Object Identifier 10.1109/JSTQE.2004.829201

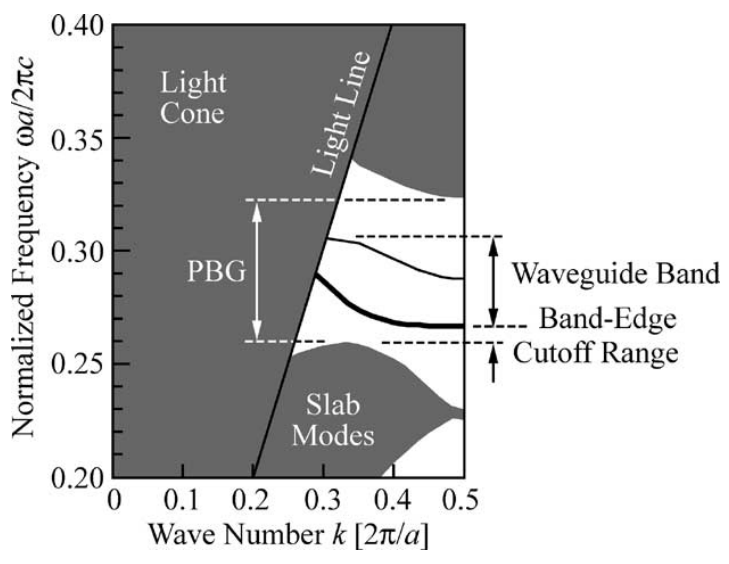

Fig. 1. Photonic band diagram of single line defect waveguide in 2-D PC of triangular lattice airholes. Polarization parallel to 2-D plane is assumed. It was calculated by the plane wave expansion method with the plane wave number of 5253. The diameter to lattice constant ratio $2 r / a$ is 0.64 . The background index is 2.73. Thick curve shows the fundamental waveguide band. Light line and light cone in an air-bridge PC slab is displayed as a reference.

leakage loss of the resonant mode. It simultaneously achieves a high $Q$ factor and a small mode volume of, e.g., $>10000$ and $<0.5(\lambda / n)^{3}$, respectively, where $\lambda$ is the resonant wavelength and $n$ the modal effective index. These values will be attractive for realizing a single-photon emitter with a response speed enhanced by the Purcell effect, and narrow band wavelength division multi/demultiplexers with a wide free spectral range. But for other applications, such a very small mode volume is not necessarily required. The stable single-mode operation with a moderate output power would rather be desired for a standard microlaser in a photonic integrated circuit. If the microcavity is used like a cavity etalon, which enhances the internal field and light-matter interaction, the enhancement in a moderately wide spectral range with the dispersion management against a short input pulse is more important.

In this paper, we discuss two types of light localizations, which are effective for the mode control in a large defect, the enhancement of internal field in a wide spectral range, and so on. They are different from the conventional point defect microcavity in the respect that they utilize a line defect PC waveguide. Fig. 1 shows a photonic band diagram of a single line defect waveguide in a 2-D PC of triangular lattice airholes. In all the results in this paper, we assume the polarization parallel to the 2-D plane, since the PC structure exhibits a wide PBG for this polarization. One important property seen in this figure is the cutoff frequency range between the band edge of the fundamental waveguide band (lower thick curve in the PBG) and the highest frequency of the lowest slab mode region. As theoretically demonstrated in [15], light is reflected at the input end of 
a PC waveguide at the cutoff range. Therefore, the cutoff range acts as a full PBG even with the line defect. If an additional defect is doped into the line defect, a mode with a resonant frequency at the cutoff range is strongly localized at the defect, while other modes overlapping with the waveguide band are radiated out through the waveguide. This concept provides wide variations in microcavity structures, and allows useful mode controls such as the single-mode operation.

The other important property seen in Fig. 1 is the band edge itself. At this point, the slope of the waveguide band, which is proportional to the group velocity $v_{g}$ of guided light, becomes zero due to the Bragg condition [16]. It forms a standing wave not localized at a particular point but widely distributing in the line defect. To realize a strong light localization around a particular point, we propose to use a chirping of the line defect. Here, the chirping means a gradual change of some structural parameters, which continuously shifts the waveguide band along the line defect. Related with this, heterostructure PCs have been studied [17]-[19], in which similar step-like changes for the lattice constant and the element size are used to form reflection boundaries in the investigation of modal behaviors and in the development of waveguides and multi/demultiplexers. The chirped waveguide is regarded as a kind of heterostructure in the broad sense. But the chirping is not limited to the above-mentioned similar change; any parameters and their combinations can be used, only if they shift the band property. In addition, we rather expect the chirped waveguide to suppress the reflection in its gradient profile so that it exhibits an overall response. Let us consider a situation that light of a certain frequency is initially guided in the waveguide. When the light approaches a point corresponding to the band-edge condition, its velocity is reduced and the light is finally localized. One may think that the structure looks similar to a chirped grating filter. However, clear light localization does not occur in a conventional chirped grating filter due to the sudden reflection and/or the scattering in lateral directions. Therefore, light localization is a unique phenomenon in a chirped PC waveguide. Since the localization occurs at different wavelengths and positions, this concept is effective for realizing the localization of a short pulse with a wide spectrum.

In Sections II and III, we describe various microcavities at the cutoff range and the light localization phenomenon in chirped waveguides, respectively. We will present their general features by showing some theoretical and experimental results and discuss their possibilities of future applications.

\section{Microcavities at Cutoff RANGe}

\section{A. Cavity Structures}

Fig. 2(a)-(c) shows three kinds of cavity structures in a 2-D PC of triangular lattice airholes. Structure (a) has a point defect put into one or multiple line defects. We call it a point and line composite defect [20]. As an example, resonant spectra and mode profiles are calculated for a composite of $\mathrm{H} 2$ point defect (hexagonal cavity of seven missing airholes) and three crossing line defects by using the finite-difference time-domain (FDTD) method, as shown in Fig. 3. Here, we considered a PC slab in a 2 -D model by assuming a background index of 2.73 as an effective index of an air-bridge slab. The analysis space was divided

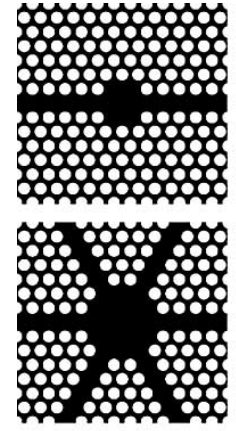

(a)

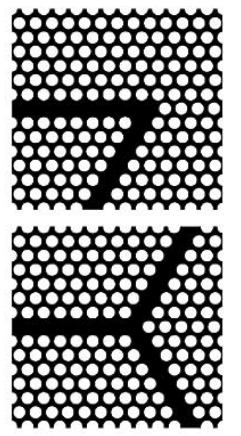

(b)

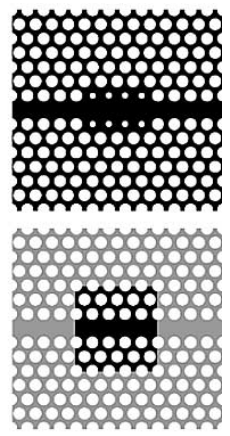

(c)
Fig. 2. Various cavity structures which utilize the cutoff range of line defect. (a) Point and line composite defects (b) Waveguide components. (c) Modified waveguides.

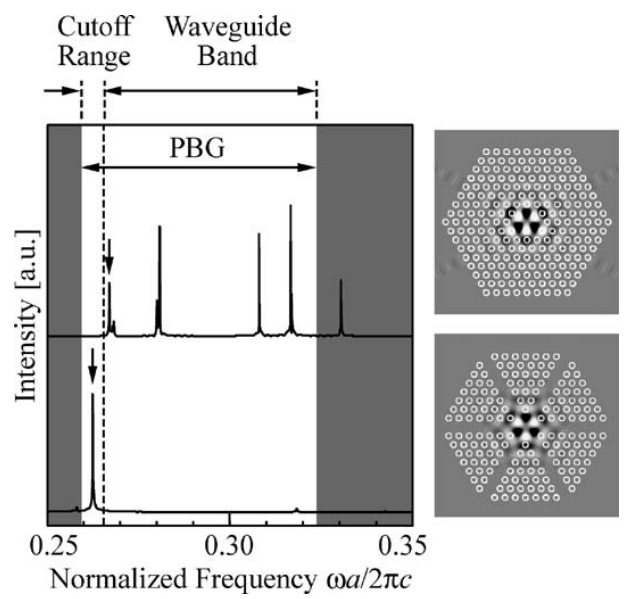

Fig. 3. Resonant spectra and field profiles for $\mathrm{H} 2$ point defect (upper) and composite of $\mathrm{H} 2$ and three crossing line defects (lower). They are calculated by the FDTD method. Calculation parameters are the same as for Fig. 1. Spectra corresponding to field profiles are indicated by arrows.

by $(\sim a / 20)^{2}$ square Yee cells, where $a$ is the lattice constant. The normalized airhole diameter $2 r / a$ was set to be 0.64 . In the simple $\mathrm{H} 2$ cavity, many resonant modes appear due to the wide PBG (the gap width is $22 \%$ of the midgap frequency) and the relatively large cavity size (an inscribed diameter is $2.8 a$ ). But in the composite defect, modes overlapping with the waveguide band are removed, and only a mode at the cutoff range (the gap width is only $4.5 \%$ of the midgap frequency) remains. The field profile of the localized mode in the composite defect is very similar to that in the $\mathrm{H} 2$ defect.

Structure (b) is a simple waveguide component such as bends and branches [21], [22]. Even though they look like a natural sequence of waveguides, they break the symmetry of a normal straight-line defect. Therefore, they act as additional point defects and maintain a mode similar to those in composite defects. Structure (c) utilizes a local modification of some structural parameters. If the modified region has a waveguide band at the cutoff range of the normal waveguide, the region can act as an additional point defect and confines a cutoff mode in the same way as the former two cases.

Since the cutoff range is much narrower than the PBG, it is easy to obtain the single-mode resonance. Even in a larger 


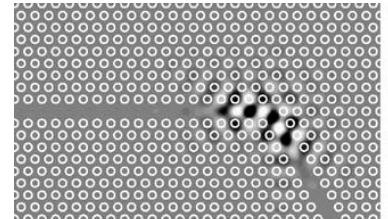

(a)

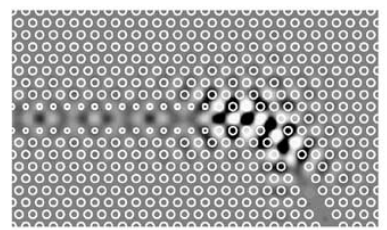

(c)

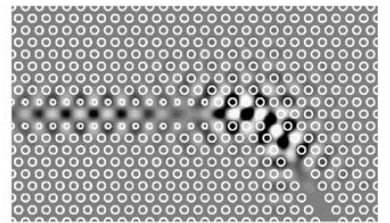

(b)

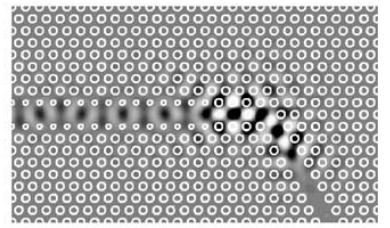

(d)
Fig. 4. FDTD simulation of light extraction from $60^{\circ}$ bend cavity to a modified waveguide. Designs of modified waveguide in (a)-(d) correspond to those in Fig. 5.

composite defect, the effective single-mode condition is maintained by adding an appropriate number of line defects. When the defect is several micrometers in diameter, an output power of milliwatt order will be expected with careful management of carriers, heat, etc. (By way of comparison, GaAs-based vertical cavity surface emitting lasers normally have milliwatt output power in a similar cavity size). For the further stabilization of the single-mode condition, one has to take care of the symmetry of the cavity structure. It was experimentally shown that a cavity structure with a highly rotational symmetry causes a multimode resonance due to the splitting of degenerate modes. For example, the smallest point defect cavity of one missing airhole in a triangular lattice PC ideally has three degenerate modes, but experimentally exhibits a multimode resonance, which is caused by the break of the degeneracy in a fabricated structure with small disordering [1]. Since bends (b) and straight structures (c) have single or double rotational symmetry, respectively, they are free from the mode degeneracy problem and achieve a more stable single-mode operation.

In addition, structures (a)-(c) are suitable for extracting light, since they are based on the PC waveguide. In these structures, a normal PC waveguide is used as a cavity mirror. By limiting the length of the normal waveguide and connecting a modified waveguide, light in the cavity region penetrates into the normal waveguide and extracted to the modified waveguide. The light extraction is controlled by the length of the normal waveguide. Fig. 4 shows the FDTD simulation of light extraction from the $60^{\circ}$ bend cavity. Here, the modified waveguide has airholes with a reduced diameter $2 r^{\prime}$ just beside the line defect. The corresponding photonic bands are shown in Fig. 5. By reducing $2 r^{\prime}$ from $2 r$, the fundamental waveguide band shifts to the lower frequency side, so the cutoff range finally disappears. The horizontal dotted line indicates the resonant frequency of a localized mode at the $60^{\circ}$ bend. When $2 r^{\prime} / 2 r=0.83$, the mode is extracted to this modified waveguide, as shown in Fig. 4(c). A peculiar light localization inside the modified waveguide in Fig. 4(b) and the zigzag propagation in Fig. 4(d) are caused by the overlap of the resonant frequency with the band edge and with two waveguide bands, respectively.

\section{B. Experiment}

In this study, some structures of Fig. 2(a) and (b) were fabricated with normal hexagonal point defects and a single line defect in a GaInAsP PC slab to demonstrate the concept of using the cutoff range. In the experiment, a GaInAsP/InP epitaxial wafer with five compressively strained quantum wells was prepared. The airhole arrays were formed by electron beam (EB) lithography and $\mathrm{Cl}_{2} / \mathrm{Xe}$ inductively coupled plasma (ICP) etching with a metal mask. A membrane structure of the PC slab was finally formed by $\mathrm{HCl}$ selective wet etching of the InP substrate. The detail of the fabrication process is the same as that in [20]. Typical thickness $t$, lattice constant $a$, and airhole diameter $2 r$ were $0.243,0.44$, and $0.25 \mu \mathrm{m}$, respectively. Cavity structures fabricated were $\mathrm{H} 1, \mathrm{H} 2, \mathrm{H} 4$, and $\mathrm{H} 7$ point defects (the number denotes the rows of missing airholes from the center defect), composite of the $\mathrm{H} 7$ point defect and three line defects, a straight-line defect, $60^{\circ}$ and $120^{\circ}$ bends, and a Y branch. Fig. 6 shows scanning electron microscope pictures of the PC slab, the $\mathrm{H} 2$ defect, and the composite defect. The maximum sidewall roughness of airholes was less than $20 \mathrm{~nm}$, and the sidewall angle $89^{\circ}-91^{\circ}$.

For all the structures, the lasing operation was obtained at room temperature by pulsed photopumping at $\lambda=0.98 \mu \mathrm{m}$. Here, the pulse width, the repetition cycle, and the focused spot diameter of the pump light were $75 \mathrm{~ns}, 500 \mu \mathrm{s}$ and 5-6 $\mu \mathrm{m}$, respectively. Light output toward the device top was collimated and focused to a multimode fiber and analyzed by an optical spectrum analyzer. Observed lasing characteristics are shown in Fig. 7, and the corresponding spectra are shown in Figs. 8 and 9. Since devices for Fig. 7(a), (b), and (c) were fabricated in three different process cycles, we cannot compare the results between figures. In Fig. 7(a), the typical threshold power of external irradiation for $\mathrm{H} 2$ and larger point defects was nearly $1.5 \mathrm{~mW}$. The corresponding effective power estimated by considering the filling factor of air, the effective mode area, the slab thickness, and an absorption coefficient of the pump light at the slab $\left(20000 \mathrm{~cm}^{-1}\right)$ was $\sim 60 \mu \mathrm{W}$. For different-sized point defects, no significant difference in threshold was observed. This is considered to be due to the large spot diameter of pump light, which completely covered these cavities, and due to the almost same threshold power density. Therefore, if the spot diameter of pump light is reduced to $2 \mu \mathrm{m}$ for the $\mathrm{H} 2$ defect, the threshold will be reduced to $0.2 \mathrm{~mW}$. This value is close to a record value for an optimized $\mathrm{H} 1$ defect [23]. Regarding the normal $\mathrm{H} 1$ defect, the threshold was as high as $4 \mathrm{~mW}$, as shown in Fig. 7(b). One main reason for this is a poor quality of the device fabricated in this process cycle. Another important reason is the lack of structural optimization, which suppresses the optical leakage from the ultrasmall cavity toward air. As a structural optimization, we tested a modified $\mathrm{H} 1$ defect with a reduced diameter of six airholes around the center defect. Fig. 7(b) clearly shows that the smaller six airholes give the lower threshold. By employing $50 \%$ diameter airholes, the threshold was reduced to $2.2 \mathrm{~mW}$. This value was the same as that for $\mathrm{H} 2$ defect fabricated simultaneously in the process cycle.

The threshold for the composite defect is slightly higher than for the point defects, as shown in Fig. 7(a). It is considered to be 


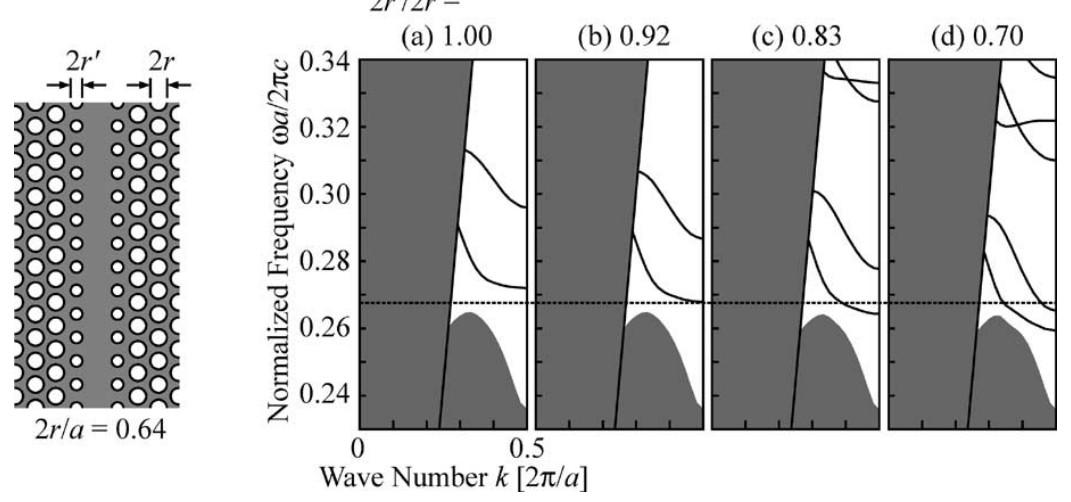

Fig. 5. Schematic of modified waveguide used for light extraction and its photonic band diagrams with different airhole diameter $2 r^{\prime}$ beside the line defect. Horizontal dotted line denotes resonant normalized frequency of $60^{\circ}$ bend cavity in Fig. 4.

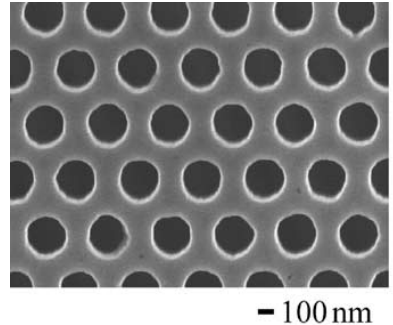

(a)

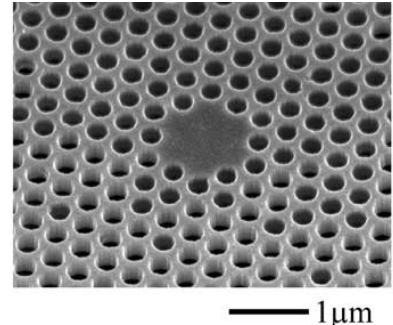

(b)

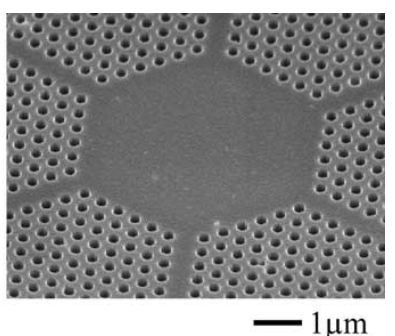

(c)

Fig. 6. Scanning electron microscope pictures of: (a) GaInAsP PC slab; (b) H2 point defect cavity; and (c) composite cavity of $\mathrm{H} 7$ point defect and three crossing line defects.

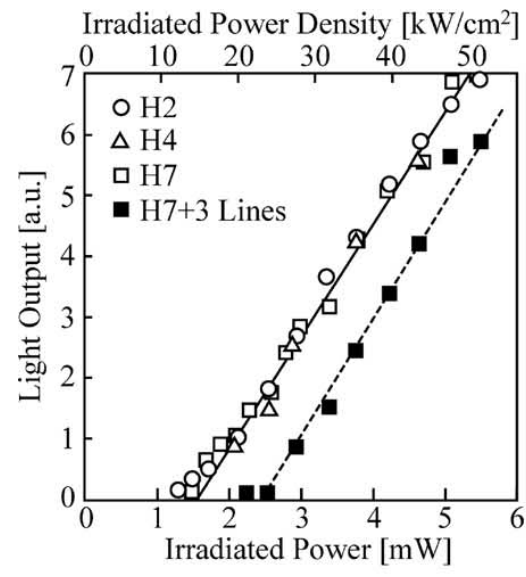

(a)

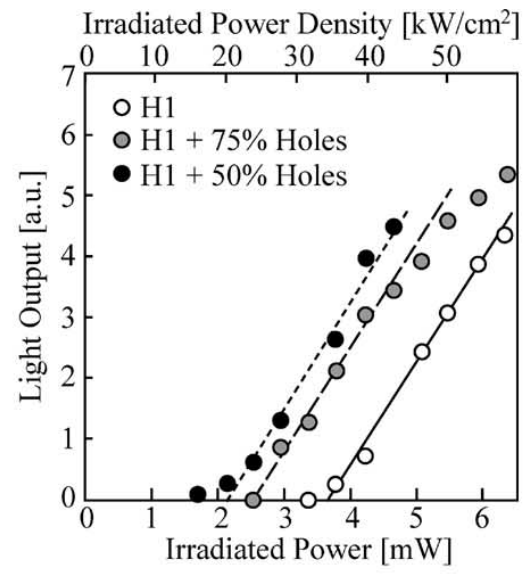

(b)

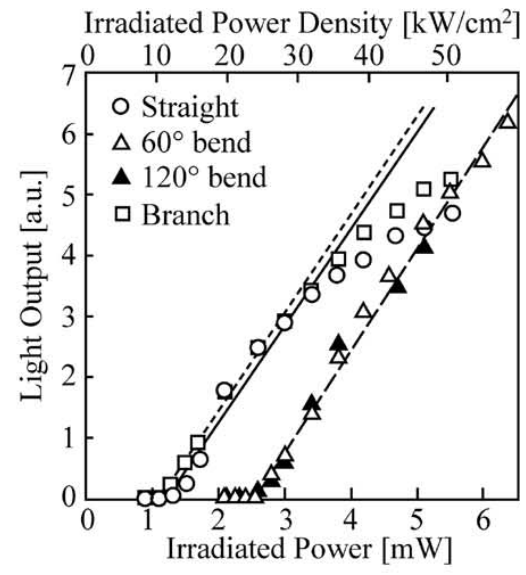

(c)

Fig. 7. Lasing characteristics at room temperature by pulsed photopumping. (a) Point and composite defect cavities. (b) H1 and its modified cavities. (c) Line, bend, and branch cavities. These three were measured for devices fabricated in three different process cycles.

due to the optical leakage and the carrier diffusion, which are enhanced by the line defects. Therefore, a structural modification for light and carrier confinement, which is a common issue for PC microcavity lasers, should be more studied for the composite defect in future. The threshold for the bends and the branch were also higher than for the point defects. The reason considered is their strongly asymmetric structures and mode profiles, which might enhance the optical leakage. This expectation is supported from the result that the triple symmetric Y-branch having a good consistency with the triangular lattice showed the lowest threshold of $\sim 1.0 \mathrm{~mW}$. The threshold for the normal straight-line defect is also measured to be as low as $1.2 \mathrm{~mW}$.
Regarding this result, however, we observed the strong position dependence; the threshold varied from 1.2 to $4.0 \mathrm{~mW}$ in different line defects and different positions. In relation to this, the lasing wavelength fluctuated by several nanometers even against the same design. Therefore, we suspect if some disordering in a line defect behaved as a composite defect cavity and generated the strong light localization. Otherwise, it is difficult to explain the position dependence and the low threshold against the small pump spot, which could not totally cover the line defect mode.

Even under such conditions, we observed a clear difference of the lasing wavelength between the straight-line defect and the waveguide components, as shown in Fig. 9. Lasing wavelengths 


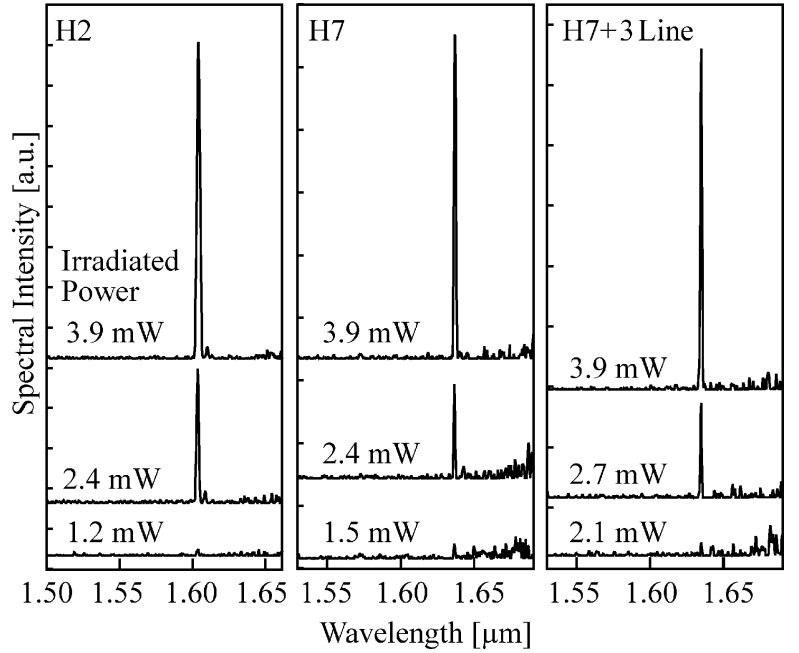

Fig. 8. Lasing spectra for point and composite defects.

for the bends and the branch were always longer than that for the straight-line defect by $10-20 \mathrm{~nm}$. This result well agrees with the FDTD simulation; the cutoff modes for the waveguide components have lower normalized frequencies than that of the band-edge mode.

\section{CHIRPED WAVEGUides}

\section{A. Structures}

Fig. 10 illustrates four examples of chirped waveguides in a 2-D PC of triangular lattice airholes, namely, chirpings of: (a) airhole diameter $2 r$; (b) lattice constant $a$; (c) both $2 r$ and $a$ with similarity; and (d) those without similarity. Let us consider the shift of photonic bands for structure (a). Fig. 11 shows band diagrams calculated in the same way as for Fig. 1. Here, the background index is assumed to be 2.963 , and $2 r / a$ is taken as a parameter. For a constant $a$, the increase in $2 r$ reduces the average refractive index of the slab, so photonic bands shift toward higher frequencies. If light of a certain frequency is initially guided through the waveguide by the fundamental waveguide band, it reaches the band-edge point by the increase. A simpler case is chirping (c) in Fig. 10. Here, the chirping changes the normalized frequency $\omega a / 2 \pi c$ in one photonic band diagram for a constant $2 r / a$. By reducing $a$, guided light simply reaches the band-edge point. On the other hand, chirpings (b) and (d) provide faster and slower changes of the waveguiding condition, respectively. In the case of (b), the decrease in $a$ simultaneously leads to the band shift to higher frequencies and the decrease in $\omega a / 2 \pi c$. Therefore, guided light reaches the band-edge point more rapidly. In the case of (d), bands shift to lower frequencies, while $\omega a / 2 \pi c$ decreases. Even under this condition, the guided light finally reaches the band-edge point by a moderate design of the chirping. But its speed is slower than others. The choice of chirp parameters depends on its purpose. Experimentally, a faster change of structural parameters is easy to fabricate. If one needs a slow approach of light to the band-edge point, (d) may be effective. However, the simultaneous control of two parameters causes another complexity in the fabrication process. For the simple fabrication, (a) must be more desirable.

\section{B. Experiment}

In this study, the structure shown in Fig. 10(a) was fabricated to observe their two characteristics arising from the chirping, i.e., the light localization at the band-edge point and the light radiation at the air light-line point. The latter phenomenon occurs when light propagates in the direction opposite to the former case. In the experiment, a unibond-type silicon-on-insulator (SOI) wafer with a top Si layer of $0.26-\mu \mathrm{m}$ thickness and an $\mathrm{SiO}_{2}$ layer of 1.0- $\mu \mathrm{m}$ thickness was used. The airhole arrays were formed by EB lithography with positive resist ZEP520 (Zeon Corporation, Louisville, $\mathrm{CO}$ ) and $\mathrm{SF}_{6} / \mathrm{O}_{2}$ ICP etching. The input end facet of the waveguide was formed by cleavage. Finally, the membrane structure of the PC slab was formed by $\mathrm{HF}$ selective wet etching of the $\mathrm{SiO}_{2}$ layer, as shown in Fig. 12(a).

The lattice constant was $0.42 \mu \mathrm{m}$, and the chirp range of the airhole diameter was $0.183-0.320 \mu \mathrm{m}$ in various waveguide lengths. The grid size of the computer-aided design (CAD) in the EB writer was $10 \mathrm{~nm}$. It was too coarse to create a sufficiently smooth chirp pattern. Therefore, we fixed the diameter to be $0.19 \mu \mathrm{m}$ in the CAD data and gradually changed the exposure time along the waveguide. By compensating the nonlinearity in airhole diameter with the exposure time, we formed an approximate linear chirping with the minimum step in diameter of $4.5 \mathrm{~nm}$. The ICP etching was carried out with the resist mask. Due to the direct transfer of the mask pattern, uniform airholes were formed, as shown in Fig. 12(b). By optimizing the gas mixture and the etching time, almost vertical sidewalls were obtained with the maximum roughness of less than $5 \mathrm{~nm}$.

In the measurement, light from a tunable laser was focused to a spot diameter of $\sim 1 \mu \mathrm{m}$ and inserted to the waveguide end. The near field pattern of light propagation was observed from the top using a vidicon camera. For the light incidence to the smaller airhole side of the waveguide, we expect that the light reaches the band-edge point and is localized. As shown in Fig. 13(a), a characteristic shift of a weak light spot was observed in a wavelength range of $1.55-1.50 \mu \mathrm{m}$. In this range, light output at the waveguide end was not clear. For shorter wavelengths, the spot reached the output end, and the strong output appeared. For the light incidence to the larger airhole side, we expect that the light reaches the light-line point and is radiated out to air. As shown in Fig. 13(a), the clear radiation beyond a particular point was observed, which shifted against the wavelength change from 1.50 to $1.47 \mu \mathrm{m}$. For some waveguide samples, the relation of $\omega a / 2 \pi c$ of input light and $2 r / a$ at positions exhibiting these two characteristic spots is summarized in Fig. 13(b). Theoretical curves for the band-edge and light-line conditions were obtained from photonic bands. Although the calculation was 2-D with the effective index approximation for the slab, the curves almost fit to experimental plots with a small adjustment of the background index to 2.75. This is the clear evidence of expected characteristics of the chirped PC waveguides.

\section{Simulation of Light Localization}

As explained above, the chirped waveguide is expected to generate light localization at the band-edge point. However, it is difficult to directly observe the microscopic behavior of light 


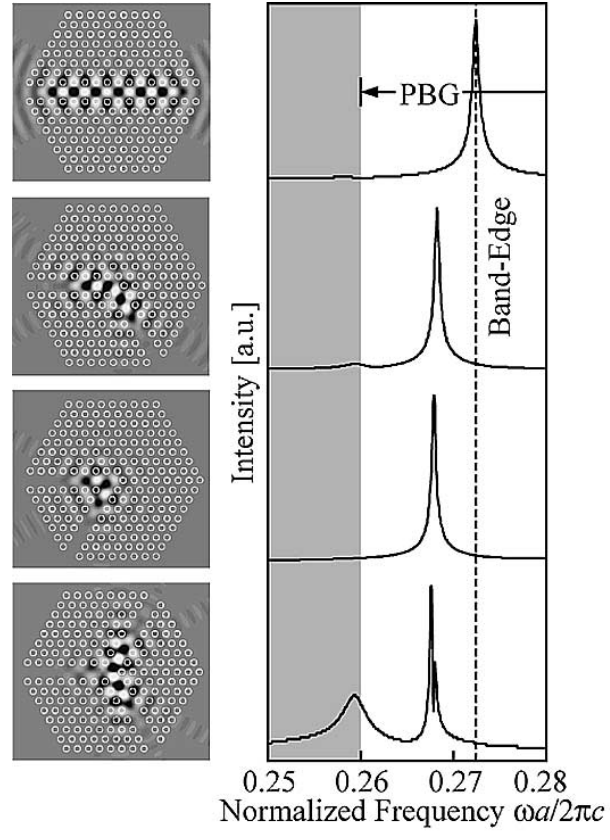

(a)

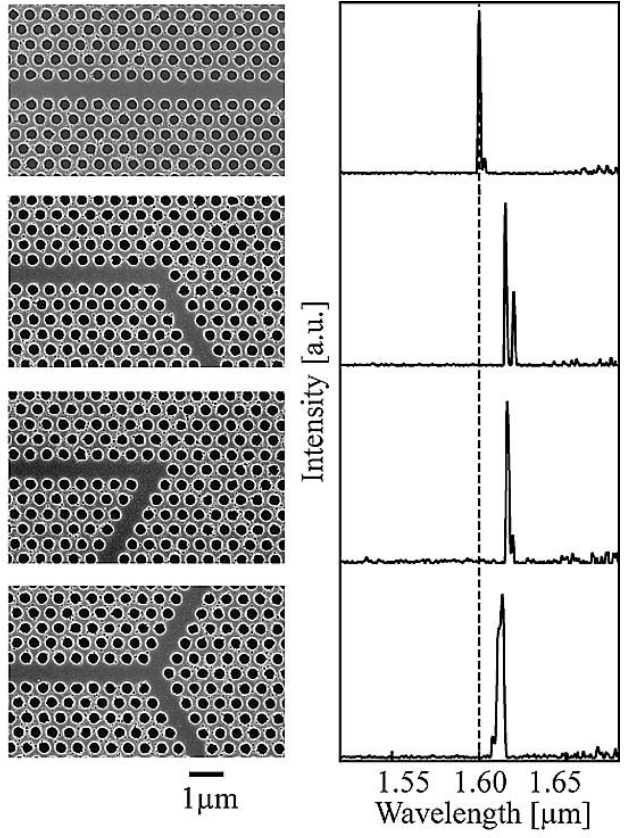

(b)

Fig. 9. Comparison of FDTD simulation and experiment for straight waveguide and waveguide components. (a) Mode profiles (magnetic field in vertical direction) and resonant spectra by the FDTD method. (b) Top views and observed lasing spectra in the experiment.

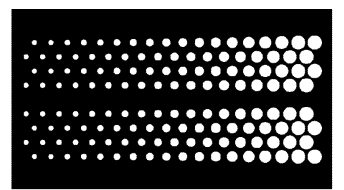

(a)

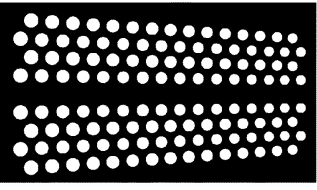

(c)

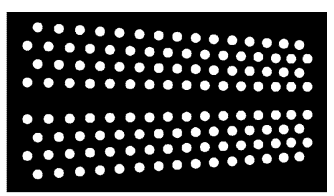

(b)

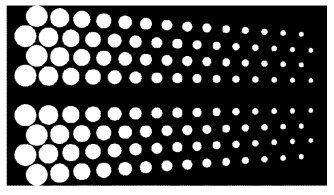

(d)
Fig. 10. Schematics of $\mathrm{PC}$ waveguide with chirping of: (a) airhole diameter $2 r$ (b) lattice constant $a$; (c) both $2 r$; and $a$ with similarity and (d) those without similarity.

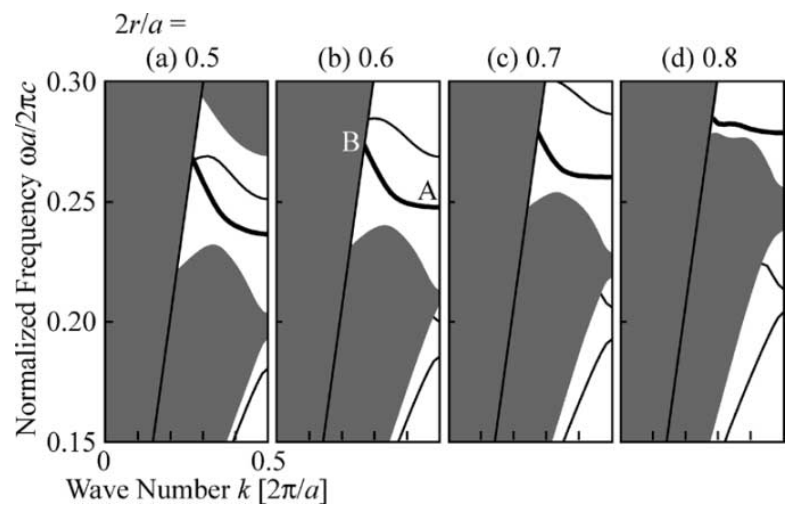

Fig. 11. Photonic band diagram of single line defect waveguide in 2-D PC of triangular lattice airholes. Method and condition of calculation are the same as for Fig. 1. The background index is assumed to be 2.963. Symbols A and $\mathrm{B}$ at ends of thick curve denote band-edge and light-line conditions of the fundamental waveguide band, respectively.

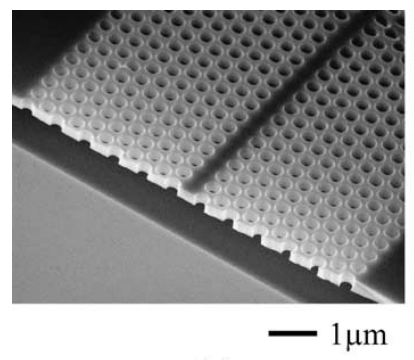

(a)

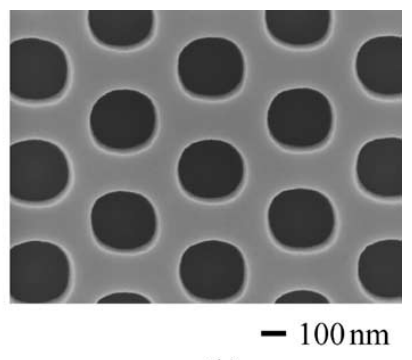

(b)
Fig. 12. Scanning electron microscope pictures of: (a) air-bridge-type single line defect PC waveguide formed into SOI substrate and (b) magnified view of $\mathrm{PC}$.

in the experiment. So we investigated this phenomenon by the FDTD simulation. We calculated the light propagation in all types of chirped waveguides in Fig. 10, and noticed that the behavior of light propagation was fundamentally the same. Fig. 14 shows the field profile of light simulated against the continuous-wave excitation in the waveguide with the airhole diameter chirping. In a series of field profiles at different time steps of the FDTD simulation, we confirmed that the guided light is decelerated and finally forms a standing wave at a particular point. As seen in Fig. 14, this point is different for different $\omega a / 2 \pi c$. The multifrequency excitation results in the multiposition localization. This localization does not mean the complete stop of optical energy at the point. Once the standing wave is formed, the increase in accumulated optical energy is saturated and the reflection starts. The reflection is explained by the finite spatial broadening of the light localization. As seen in Fig. 14, the localized field distributes at least in a length of $5 a$ and further penetrates into both sides. This suggests that the field partly exceeds the band-edge point and reflected. As a result, the macro- 


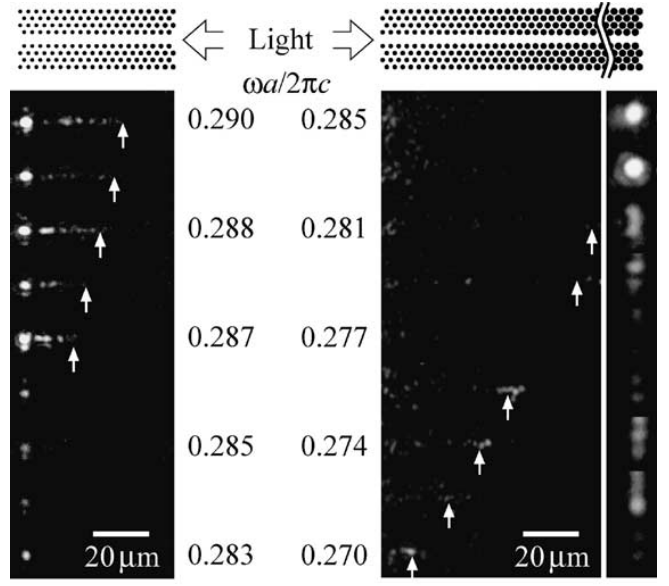

(a)

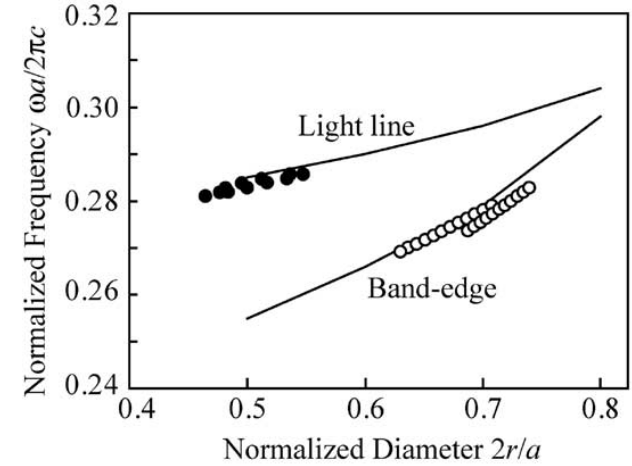

(b)

Fig. 13. Observation of light propagation in airhole diameter chirped PC waveguide and its correspondence to a theoretical result. (a) Top views of near field pattern of light incident to the waveguide from different directions. (b) Relation between normalized frequency of input light and normalized diameter exhibiting characteristic light spots. Plots and curves denote experimental results and theoretical ones obtained from photonic bands.

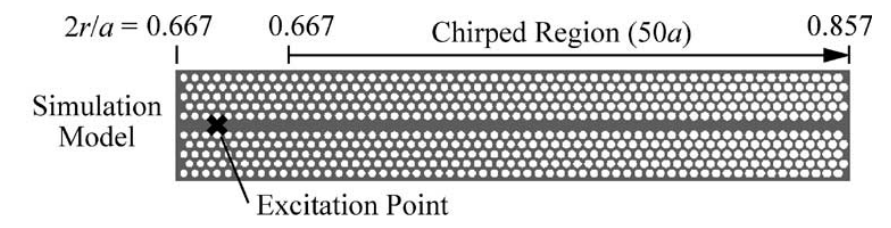

$\omega a / 2 \pi c=$

0.2625

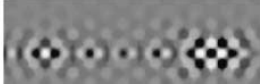

$0.263,0.266$

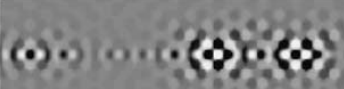

$0.263,0.266$

0.269

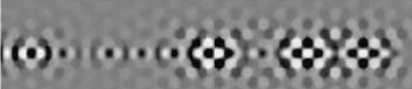

$0.263,0.266$ $0.269,0.272$

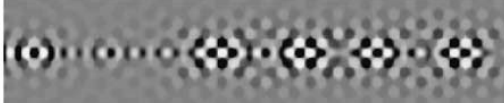

Fig. 14. FDTD simulation model of airhole diameter chirped PC waveguide and field profiles for different normalized frequencies obtained for continuouswave excitation.

scopic behavior of light in the waveguide is similar to that in a chirped grating filter, except the time delay for the formation of the standing wave.

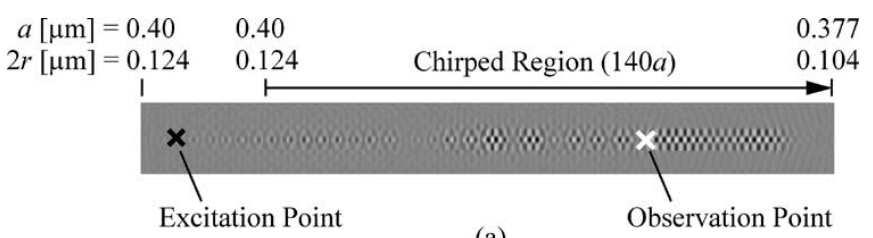

(a)

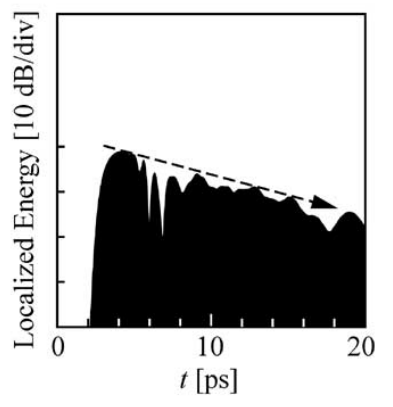

(b)

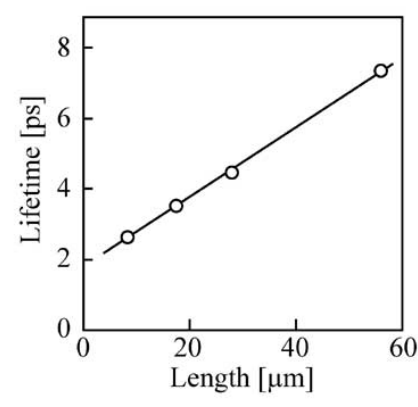

(c)
Fig. 15. Behavior of light localization against short input pulse. (a) Field profile (magnetic field in vertical direction) in chirped waveguide type (d) in Fig. 10. Horizontal distribution of the field is compressed to 60\%. (b) Time response of accumulated optical energy (square of the magnetic field), which is measured at white cross point in (a). (c) Lifetime of the decay observed in (b) as a function of total length of light localization. The length is simply controlled by changing the waveguide length with keeping chirp range of parameters.

An expectation suggested from the result is if the localization can be used for the temporary accumulation of optical energy. This expectation was tested in the simulation with a Gaussian pulse excitation, as shown in Fig. 15(a). Here, the chirped waveguide is type (d) in Fig. 10. The excited pulse has a spectral full width at $1 / e$ maximum of $1.5 \%$ of the center frequency (corresponding center wavelength is $1.55 \mu \mathrm{m})$. Due to the spectral broadening, the localized field distributes in a wide area. Its envelope profile approximately corresponds to the spectrum of the input pulse with a frequency-dependent time delay. Once the localization is completed, the accumulated energy exhibits a slow decay by the reflection. The temporal increase or decrease in the wave form may be caused by the interference between neighboring localized fields. Note that the lifetime of the decay is simply proportional to the length of light localization, as shown in Fig. 15(b). A long waveguide with a slow chirp function exhibits a long light localization and realizes a long lifetime of the accumulated energy. A nanosecond order lifetime will be obtained with a waveguide length of $1 \mathrm{~cm}$. When the spectral broadening is suppressed to $0.065 \%$ of the center frequency, which corresponds to a wavelength broadening of $1 \mathrm{~nm}$ at $\lambda=1.55 \mu \mathrm{m}$, this waveguide length will be reduced to $<500 \mu \mathrm{m}$.

\section{CONCLUSION}

We theoretically and experimentally demonstrated two different types of light localization, which are both unique in PC line defect waveguides. The light localization utilizing the cutoff frequency range provides a variety of cavity structures such as a composite defect, a waveguide component, a modified waveguide, and so on. We observed the lasing action in some of them fabricated into a GaInAsP PC slab at room temperature. We confirmed the lasing in the cutoff range from the comparison of lasing wavelengths between different cavities. 
The light localization in a chirped PC waveguide was suggested through the observation of light propagation in those fabricated into an SOI substrate. The FDTD simulation showed the frequency-dependent localization, which allows the slow decay of the accumulated optical energy. This phenomenon is expected to realize a group delay device or a dispersion control device, whose detail will be investigated with the dispersion management in the next issue.

\section{REFERENCES}

[1] O. J. Painter, A. Husain, A. Scherer, J. D. O’Brien, I. Kim, and P. D. Dapkus, "Room temperature photonic crystal defect lasers at near-infrared wavelengths in InGaAsP," J. Lightwave Technol., vol. 17, pp. 2082-2088, Nov. 1999.

[2] J. K. Hwang, H. Y. Ryu, D. S. Song, I. Y. Han, H. W. Song, H. K. Park, Y. H. Lee, and D. H. Jang, "Room-temperature triangular-lattice twodimensional photonic band gap lasers operating at $1.54 \mu \mathrm{m}$, , Appl. Phys. Lett., vol. 76, pp. 2982-2984, 2000.

[3] P. T. Lee, J. R. Cao, S. J. Choi, Z. J. Wei, J. D. O'Brien, and P. D Dapkus, "Room-temperature operation of VCSEL-pumped photonic crystal lasers," IEEE Photon. Technol. Lett., vol. 14, pp. 435-437, Apr. 2002.

[4] C. Monat, C. Seassal, X. Letartre, P. Viktorovitch, P. Regreny, M. Gendry, P. Rojo-Romeo, G. Hollinger, E. Jalaguier, S. Pocas, and B. Aspar, "InP 2D photonic crystal microlasers on silicon wafer: room temperature operation at $1.55 \mu \mathrm{m}$," Electron. Lett., vol. 37, pp. 764-765, 2001

[5] S. Noda, A. Chutinan, and M. Imada, "Trapping and emission of photons by a single defect in a photonic bandgap structure," Nature, vol. 407, no. 6084, pp. 608-610, Oct. 2000.

[6] C. J. M. Smith, R. M. DeLaRue, and M. Rattier et al., "Coupled guide and cavity in a two-dimensional photonic crystal," Appl. Phys. Lett., vol. 78, no. 11, pp. 1487-1489, Mar. 2001.

[7] S. Y. Lin, E. Chow, S. G. Johnson, and J. D. Joannopoulos et al., "Direct measurement of the quality factor in a two-dimensional photonic-crystal microcavity," Opt. Lett., vol. 26, no. 23, pp. 1903-1905, Dec. 2001.

[8] T. Baba, N. Fukaya, and J. Yonekura, "Observation of light transmission in photonic crystal waveguides with bends," Electron. Lett., vol. 35, pp. 654-655, 1999.

[9] M. Tokushima, H. Kosaka, A. Tomita, and H. Yamada, "Lightwave propagation through a 120 degrees sharply bent single-line-defect photonic crystal waveguide," Appl. Phys. Lett., vol. 76, pp. 952-954, 2000.

[10] M. Lončar, D. Nedeljković, T. Doll, J. Vučković, A. Scherer, and T. P. Pearsall, "Waveguiding in planar photonic crystals," Appl. Phys. Lett., vol. 77, pp. 1937-1939, 2000

[11] S. Y. Lin, E. Chow, S. G. Johnson, and J. D. Joannopoulos, "Demonstration of highly efficient waveguiding in a photonic crystal slab at the 1.5- $\mu$ m wavelength," Opt. Lett., vol. 25, pp. 1297-1299, 2000.

[12] C. J. Smith, H. Benisty, S. Olivier, M. Rattier, C. Weisbuch, T. F. Krauss, R. M. De La Rue, R. Houdré, and U. Oesterle, "Low-loss channel waveguides with two-dimensional photonic crystal boundaries," Appl. Phys. Lett., vol. 77, pp. 2813-2815, 2000.

[13] M. Notomi, A. Shinya, K. Yamada, J. Takahashi, and I. Yokohama, "Single-mode transmission within photonic bandgap of width-varied single-line-defect photonic crystal waveguides on SOI substrates," Electron. Lett., vol. 37, pp. 293-295, 2001.

[14] Y. Sugimoto, N. Ikeda, N. Carlsson, and K. Asakawa, "AlGaAs-based two-dimensional photonic crystal slab with defect waveguides for planar lightwave circuit applications," IEEE J. Quantum Electron., vol. 38, pp. 760-769, July 2002.

[15] J. Yonekura, M. Ikeda, and T. Baba, "Analysis of finite 2-D photonic crystals of columns and lightwave devices using the scattering matrix method," J. Lightwave Technol., vol. 17, pp. 1500-1508, Aug. 1999.

[16] M. Notomi, K. Yamada, A. Shinya, J. Takahashi, C. Takahashi, and I. Yokohama, "Extremely large group velocity dispersion of line-defect waveguides in photonic crystal slabs," Phys. Rev. Lett., vol. 87, p. 253 902, 2001.

[17] S. Yano, Y. Segawa, and J. S. Bae, "Quantized state in a single quantum well structure of photonic crystals," Phys. Rev. B, vol. 6315, pp. 3316-3320, 2001
[18] Y. Ohtera, T. Kawashima, Y. Sakai, and S. Kawakami, "Photonic crystal waveguides utilizing a modulated lattice structure," Opt. Lett., vol. 27, pp. 2158-2160, 2002.

[19] B. S. Song, S. Noda, and T. Asano, "Photonic devices based on in-plane hetero photonic crystals," Science, vol. 300, p. 1537, 2003.

[20] K. Inoshita and T. Baba, "Fabrication of GaInAsP/InP photonic crystal lasers by ICP etching and control of resonant mode in point and line composite defects," IEEE J. Select. Topics Quantum Electron., vol. 9, pp. 1347-1354, Oct. 2003

[21] _ , "Lasing at bend, branch and intersection of photonic crystal waveguides," Electron. Lett., vol. 39, pp. 844-846, 2003.

[22] — , "Room temperature lasing characteristics of bend and branch in photonic crystal waveguides," Jpn. J. Appl. Phys., vol. 42, pp. 6887-6891, 2003.

[23] M. Lončar, T. Yoshie, A. Scherer, P. Gogna, and Y. Qiu, "Low-threshold photonic crystal laser," Appl. Phys. Lett., vol. 81, pp. 2680-2682, 2002.

Toshihiko Baba (M'93) was born in Nagano Prefecture, Japan, on November 12, 1962. He received the B.E., M.E. and Ph.D. degrees from the Division of Electrical and Computer Engineering, Yokohama National University (YNU), in 1985, 1987, and 1990, respectively. His Ph.D. work involved antiresonant reflecting optical waveguides (ARROWs) and lightwave circuits.

From 1990 to 1994, he was a Research Associate at the Tokyo Institute of Technology, where he worked on the spontaneous emission behavior in vertical cavity surface emitting lasers (VCSELs) and achieved the room temperature continuous-wave (RT-CW) operation of a long-wavelength VCSEL. In 1994, he became an Associate Professor with YNU and started research on photonic crystals (PCs) and microdisk lasers (MDLs). Regarding PCs, he reported the first fabrication and characterization of an InP-based light emitter and a line defect waveguide at lightwave frequencies. He also studied an enhancement of light extraction efficiency and passivation techniques Regarding MDLs, he achieved the first RT-CW operation with the smallest cavity and the lowest threshold, discussed some modifications of the cavity structure into microgear or PC-like structure and demonstrated a MDL-based near field sensor. Besides these, he is also active on a deep grating distributed Bragg reflector for short-cavity lasers and Si photonics based on nanowire waveguides. His recent interests are various cavities, lasers, and functional devices.

Dr. Baba is a Member of the Institute of Electronics, Information and Communication Engineers (IEICE), the Japan Society of Applied Physics, and the American Physics Society. He received the Niwa Memorial Prize in 1991, the Best Paper Award of Micro-Optic Conference in 1993 and 1999, the Paper Award and Academic Encouragement Award from IEICE in 1994, and the Marubun Research Encouragement Award in 2000.

Daisuke Mori was born in Kanagawa, Japan, on August 16, 1979. He received the B.E. degree from the Division of Electrical and Computer Engineering, Yokohama National University (YNU), in 2003. He is currently working toward the M.E. degree at YNU.

Mr. Mori is a Member of the Japan Society of Applied Physics (JSAP).

Kyoji Inoshita was born in Kagawa, Japan, on June 11, 1974. He received the B.E., M.E., and Ph.D. degrees from the Division of Electrical and Computer Engineering, Yokohama National University (YMU), in 1999, 2001, and 2004, respectively. His Ph.D. work involved photonic crystal microlasers.

He is currently with the Sanyo Electric Co., Ltd., Osaka, Japan.

Dr. Inoshita is a Member of the Japan Society of Applied Physics (JSAP).

Yusuke Kuroki was born in Miyazaki, Japan, on December 7, 1981. He received the B.E. degree from the Division of Electrical and Computer Engineering, Yokohama National University (YNU), in 2004. He is currently working toward the M.E. degree at YNU.

Mr. Kuroki is a member of the Japan Society of Applied Physics (JSAP). 